\title{
Hardware Considerations for Preclinical Magnetic Resonance of the Kidney
}

\author{
Paula Ramos Delgado, Ekkehard Küstermann, André Kühne, \\ Jason M. Millward, Thoralf Niendorf, Andreas Pohlmann, \\ and Martin Meier
}

\begin{abstract}
Magnetic resonance imaging (MRI) is a noninvasive imaging technology that offers unparalleled anatomical and functional detail, along with diagnostic sensitivity. MRI is suitable for longitudinal studies due to the lack of exposure to ionizing radiation. Before undertaking preclinical MRI investigations of the kidney, the appropriate MRI hardware should be carefully chosen to balance the competing demands of image quality, spatial resolution, and imaging speed, tailored to the specific scientific objectives of the investigation. Here we describe the equipment needed to perform renal MRI in rodents, with the aim to guide the appropriate hardware selection to meet the needs of renal MRI applications.

This publication is based upon work from the COST Action PARENCHIMA, a community-driven network funded by the European Cooperation in Science and Technology (COST) program of the European Union, which aims to improve the reproducibility and standardization of renal MRI biomarkers. This chapter on hardware considerations for renal MRI in small animals is complemented by two separate publications describing the experimental procedure and data analysis.
\end{abstract}

Key words Magnetic Resonance Imaging (MRI), Kidney, Mice, Rats, Hardware, RF coils, Renal MRI

1 Introduction

The detection, staging, and monitoring of kidney diseases (KD) using measurements of renal function and structure is critical in clinical nephrology. The use of animal models in kidney research promotes the understanding of disease and therapy mechanisms. While current imaging modalities such as computed tomography or ultrasound can provide some information on structural changes in the kidney, these approaches offer limited insights into functional changes.

Magnetic resonance imaging (MRI) is a noninvasive imaging technology that offers an unparalleled level of anatomical and functional detail, along with diagnostic sensitivity. MRI is suitable 
for longitudinal studies due to the lack of exposure to ionizing radiation. Together with established imaging techniques, novel approaches such as dynamic contrast-enhanced MRI (DCE-MRI), arterial spin labeling (ASL), blood oxygen level dependent MRI (BOLD), and diffusion-weighted imaging (DWI) can provide a broad characterization of the kidney, including renal vascular perfusion, oxygenation, and glomerular filtration rate.

For performing dedicated MR measurements of the kidney, the appropriate MRI hardware should be carefully chosen to balance the competing demands of image quality, spatial resolution and imaging speed, tailored to the specific scientific objectives of the investigation. This chapter is intended to outline the key hardware components of an MRI scanner and to provide an overview of currently established technologies, with the goal to guide the appropriate hardware selection that meets the needs of preclinical MRI of the kidney.

This chapter on hardware considerations for renal MRI in small animals is part of the book Pohlmann A, Niendorf T (eds) (2020) Preclinical MRI of the Kidney-Methods and Protocols. Springer, New York.

\section{Static Magnetic Field Strength $\left(B_{0}\right)$}

A superconducting magnet that generates a constant homogeneous magnetic field is the core hardware component of an MRI scanner. This strength of this static magnetic field $\left(\mathrm{B}_{0}\right)$ is proportional to the net polarization produced within the sample. The detected MR signal on a receive RF coil depends on $\mathrm{B}_{0}{ }^{2}$, whereas the noise increases linearly with $\mathrm{B}_{0}[\mathrm{l}] . \mathrm{B}_{0}$ governs the signal-to-noise ratio (SNR), which is the currency for MR image quality. This provides strong motivation and momentum to move to increasingly higher magnetic field strengths for preclinical and clinical renal MRI applications [2-4]. The proportionality of SNR with $\mathrm{B}_{0}$ is linear in the quasi-static regime, as demonstrated theoretically $[5,6]$ and experimentally [7]. For higher magnetic field strengths, wave propagation and the increasing resonance frequencies must be considered [2]. Additionally, an experimental study on intrinsic SNR behaviour with increasing $B_{0}$ field strengths $(3.0,7.0$, and $9.4 \mathrm{~T})$ in the human brain reported a superlinear increase in $\mathrm{SNR}\left(\mathrm{SNR} \propto \mathrm{B}_{0}^{1.65}\right)$ [7]. This observation was also confirmed in a simulation study [8].

Relaxation time constants of biological tissues also change with the magnetic field strength, thus influencing the SNR as well as the contrast-to-noise ratio (CNR), which ultimately has an impact on the image quality. The longitudinal relaxation time $\left(T_{1}\right)$ increases nonlinearly with increasing $B_{0}[1,9-11]$. The transversal relaxation $\left(\mathrm{T}_{2}\right)$ and $\mathrm{T}_{2}{ }^{*}$ decrease at different slopes linearly $[1,9,12-$ 14]. These dependencies on $B_{0}$ are responsible for the reduced 
$\mathrm{T}_{1}$-weighted contrast and increased BOLD effect at higher magnetic field strengths ( see Note 1 ). MRI methods based on $\mathrm{T}_{2}{ }^{*}$ weighting (e.g., susceptibility weighted imaging, BOLD) will benefit from the $T_{2}{ }^{*}$ shortening, and thus increase in sensitivity at high magnetic field strengths, which underlies their broad application in renal MRI. An example of renal $T_{2}{ }^{*}$-mapping $\left(R_{2}^{*}=1 / T_{2}^{*}\right)$ is the study of oxygenation and haemodynamics in renal disorders (for a review see [15]). MR spectroscopy also benefits from the enhanced signal dispersion achieved at higher magnetic field strengths, which results in better resolved spectra, and permits improved accuracy of metabolite identification and quantification $[1,16]$.

However, MRI at high magnetic field strengths is susceptible to more severe $\mathrm{B}_{0}$ and $\mathrm{B}_{1}$ inhomogeneities, compared to lower field strengths. These inhomogeneities are primarily due to differences in the magnetic susceptibility at tissue interfaces. Susceptibility artifacts present themselves as $\mathrm{T}_{2}{ }^{*}$-induced signal losses (or even signal voids) in areas with very high $\mathrm{B}_{0}$ gradients, low phaseencoding bandwidth-related image distortion and off-resonance effects caused by $\Delta \mathrm{B}_{0}$-induced frequency dispersions. MRI of the kidney poses additional challenges due to the anatomical context. The kidneys are adjacent to bowels, in close proximity to skin/fat/ muscle boundaries (e.g., perirenal fat) or in areas containing cavities might be particularly prone to susceptibility artifacts.

Artifacts are visible as signal losses at interfaces between fat and tissue or air and tissue (susceptibility artifacts), image blurring, image distortion, and broadening of spectral lines in MRS, which interferes with the reliable differentiation of metabolites. $\mathrm{B}_{0}$ inhomogeneities can be mitigated using $\mathrm{B}_{0}$ shimming (see Subheading 3 ).

$B_{1}$ inhomogeneities lead to variations in the flip angle driven by the RF pulse excitation. $B_{1}$ inhomogeneities affect tissue contrast and SNR, appearing as signal intensity variations or losses across the field of view. Nonuniformities in $B_{1}$ are caused by the RF coil (e.g., surface RF coil, see Subheading 4) or by the creation of standing waves and constructive/destructive interferences within the object under investigation, due to the reduction of the effective wavelength which at higher field strengths becomes shorter, and can approach the dimensions of the specific anatomical structures under study [17]. While the RF wavelength inside tissue at $3.0 \mathrm{~T}$ $(\sim 128 \mathrm{MHz})$ is about $30 \mathrm{~cm}$, at $7.0 \mathrm{~T}(\sim 300 \mathrm{MHz})$ it is reduced to about $13 \mathrm{~cm} \mathrm{[18]} \mathrm{and} \mathrm{to} 6.5 \mathrm{~cm}$ at $14.0 \mathrm{~T}(\sim 600 \mathrm{MHz})$. In preclinical renal MRI, this is not a major concern, as the size of the abdomen in small animals is typically much smaller than the RF wavelength.

RF power requirements for spin excitation increase quadratically with $\mathrm{B}_{0}$. This imposes major concerns, especially for human applications, since the RF power deposition can easily reach the regulatory limits specified by IEC guidelines for the specific 
2.1 SNR Comparison for Magnetic Field Strength absorption rate (SAR) [16]. This constraint can be adressed by modification and adaptation of MR pulse sequences, acquisition strategies, and RF coils [18, 19]. In the case of preclinical studies, these issues have received less attention since there are no enforced SAR guidelines for small animals. Nevertheless, the physiological effects of tissue heating due to heavy RF deposition in experimental animals could be a serious confounding factor in preclinical studies that should not be ignored.

The SNR gains resulting from increasing $\mathrm{B}_{0}$, field have been computationally estimated for brain MRI to range from $\mathrm{B}_{0}{ }^{1.2}$ (close to the brain surface) up to $\mathrm{B}_{0}^{2.1}$ (at the center of the head) [8]. Pohmann et al. [7] investigated the field dependence of SNR for magnetic field strengths ranging between $3.0 \mathrm{~T}$ and $9.4 \mathrm{~T}$ using gradient echo techniques, and reported that SNR increases superlinearly $\left(\mathrm{B}_{0}{ }^{\mathrm{l} .65}\right)$. Using this relationship, the calculated relative SNR (normalized for the typical case of a $3.0 \mathrm{~T}$ scanner) and resolution gains (in $\mu \mathrm{m}$, in-plane resolution $1 \mathrm{~mm}$ ) corresponding to all static magnetic field strengths from $1.5 \mathrm{~T}$ to $21.1 \mathrm{~T}$ are shown in Table 1.

The increase in SNR provided by higher magnetic field strengths can be invested in enhanced spatial resolution or improvements in imaging speed. The triad of $\mathrm{B}_{0}$, spatial resolution, and imaging speed form the so-called SNR conundrum in which the competing constraints of SNR, CNR, spatial resolution, and imaging speed govern the image quality. For example, increasing the matrix size for a certain field of view will increase the spatial resolution-which will permit the visualization of more subtle anatomical structures in the kidney-but at a cost of reducing the SNR, or signal intensity per pixel/voxel. Although resolution in the frequency-encoding direction can be conveniently enhanced by increasing the matrix size without major scan time penalty, increasing spatial resolution along the phase-encoding direction substantially prolongs the scan time. Boosting the SNR is also possible by modifying the size of the field of view or the slice thickness: the larger these parameters are, the more signal is acquired. Another way to further increase SNR is signal averaging, which reduces the noise but comes at the cost longer scan times. Depending on the individual experimental context, longer scan times may be a reasonable price to pay (especially for ex vivo studies). For in vivo studies, animal welfare considerations call for the minimum scan time possible to achieve the scientific objective, and shorter scans promote higher throughput of individual animals to improve the experimental sample size.

Additional strategies to gain SNR include the use of SNR-promoting rapid imaging techniques such as RARE imaging, and dedicated RF coil technology, including cryogenically cooled RF coils (see Subheading 4). 
Table 1

Relative SNR and resolution gain with increasing static magnetic field strength

\begin{tabular}{|lll}
\hline Magnetic field, $\mathbf{B}_{\mathbf{0}}(\mathbf{T})$ & Relative SNR gain & Resolution gain (in-plane $\mathbf{1} \mathbf{~ m m})\left(\mathbf{u m}^{\mathbf{2}}\right)$ \\
\hline 1.5 & 0.3 & $(1550 \times 1550)$ \\
\hline 3.0 & 1.0 & $(495 \times 495)$ \\
\hline 4.7 & 2.1 & $(235 \times 235)$ \\
\hline 7.0 & 4.0 & $(122 \times 122)$ \\
\hline 9.4 & 6.6 & $(75 \times 75)$ \\
\hline 11.7 & 9.4 & $(52 \times 52)$ \\
\hline 14.0 & 12.7 & $(39 \times 39)$ \\
\hline 16.4 & 16.5 & $(30 \times 30)$ \\
\hline 17.6 & 18.5 & $(27 \times 27)$ \\
\hline 21.1 & 25.0 & $(20 \times 20)$ \\
\hline
\end{tabular}

SNR values were computed as proportional to $\mathrm{B}^{1.65}$. The relative SNR gain was calculated relative to that of $3.0 \mathrm{~T}$

2.2 Magnetic Field

Strengths Used in Recently Published Studies
To provide guidance as to which $\mathrm{B}_{0}$ is necessary or sufficient for preclinical MRI of the kidney, a total of 121 publications published over the past 10 years were reviewed. Of these, 66 publications reported on renal MRI in rats, and 55 in mice. Figure 1 shows the number of publications found per magnetic field strength for renal MRI in rats and mice. In rats, a total of 25 publications $(38 \%)$ were performed at $3.0 \mathrm{~T}$, closely followed by $9.4 \mathrm{~T}$ with 20 papers $(30 \%)$. In mice the preferred magnetic field strength was $7.0 \mathrm{~T}(25$ publications, $46 \%$ ). The magnetic field strengths of $9.4 \mathrm{~T}$ (8 papers, $15 \%), 3.0 \mathrm{~T}$ (7 papers, $13 \%)$, and $11.7 \mathrm{~T}$ ( 6 publications, $11 \%)$ were also used for MRI studies of mouse kidney.

Figure 2 illustrates the number of publications per magnetic field strength, listed by year for rats and mice. In rats, the number of studies performed at 3.0 and $9.4 \mathrm{~T}$ was quite stable for the last 7 years. Studies at $4.7 \mathrm{~T}$ (7 papers, 11\%) were also widely reported, as shown in the trends displayed since 2009 . The use of field strengths of 1.5 and $7.0 \mathrm{~T}$ (7 and 6 publications, respectively) varied during the period under investigation. In contrast, $7.0 \mathrm{~T}$ was established as the typical magnetic field strength for preclinical MRI of the kidney in mice during this period. In the last few years, 11.7 $\mathrm{T}$ and 16.4 Thave also emerged as MR systems suitable for the study of the mouse kidney. 


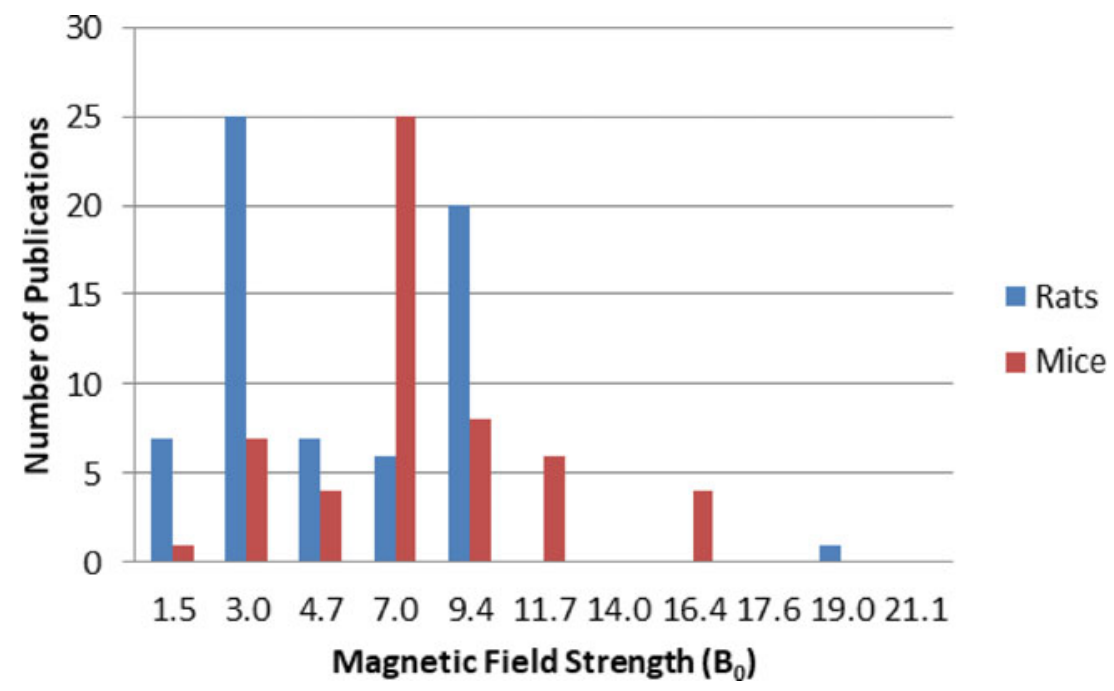

Fig. 1 Number of publications per magnetic field strength for renal MRI in rats and mice, within a total of 121 reports on preclinical renal MRI published in the past 10 years

\section{Gradient Systems}

To enable the spatial localisation of the MR signal, magnetic field gradients are used, which are provided by gradient coils. Gradient coils produce a linear variation of the magnetic field as a function of the spatial position (X-, Y-, and Z-direction) modifying the resonance frequency for spatial encoding of the MR signal.

Typical parameters governing the performance of the gradient system are the peak gradient strength, the rise time, and the slew rate. The peak gradient strength is the maximum achievable gradient strength (in $\mathrm{mT} / \mathrm{m}$ ). Typical peak gradient amplitudes in preclinical scanners are in the range of $200-1000 \mathrm{mT} / \mathrm{m}$. Clinical scanners used for preclinical MRI of the kidney provide maximum gradient amplitudes ranging between $45 \mathrm{mT} / \mathrm{m}$ and $82 \mathrm{mT} / \mathrm{m}$. The gradient rise time is the time needed to change the gradient field from zero to the peak amplitude (in ms). The gradient slew rate is the most important parameter, and refers to the speed at which a gradient can be switched. It is defined as the peak gradient strength divided by the gradient rise time (in $\mathrm{T} / \mathrm{m} / \mathrm{s}$ ). This defines the maximum scanning speed, since it influences the minimum achievable repetition time (TR) and echo time (TE), as well as the echo spacing in fast imaging techniques such as RARE or EPI (see Note 2). Typical slew rates in preclinical scanners are in the range of 640-9000 T/m/s. Clinical scanners used for preclinical MRI of the kidney offer slew rates ranging between $150 \mathrm{~T} / \mathrm{m} /$ $\mathrm{s}$ and $200 \mathrm{~T} / \mathrm{m} / \mathrm{s}$. Gradient coils can be driven in two modes: (a) constant rise time, in which the slew rate is adapted for each gradient amplitude and (b) maximum slew rate, where the rise is adjusted for each gradient amplitude. 
Rats
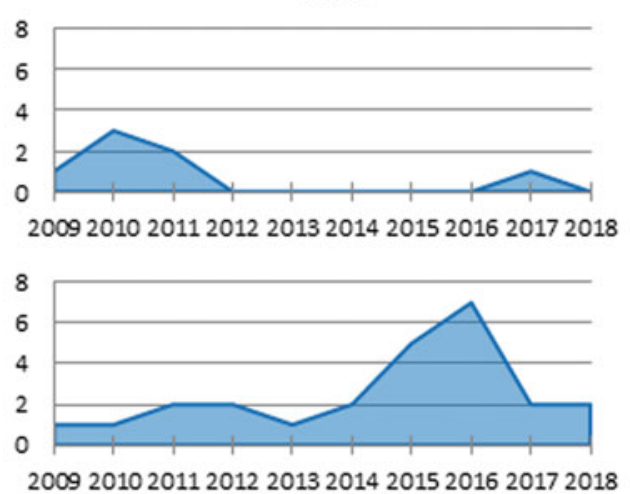

2009201020112012201320142015201620172018

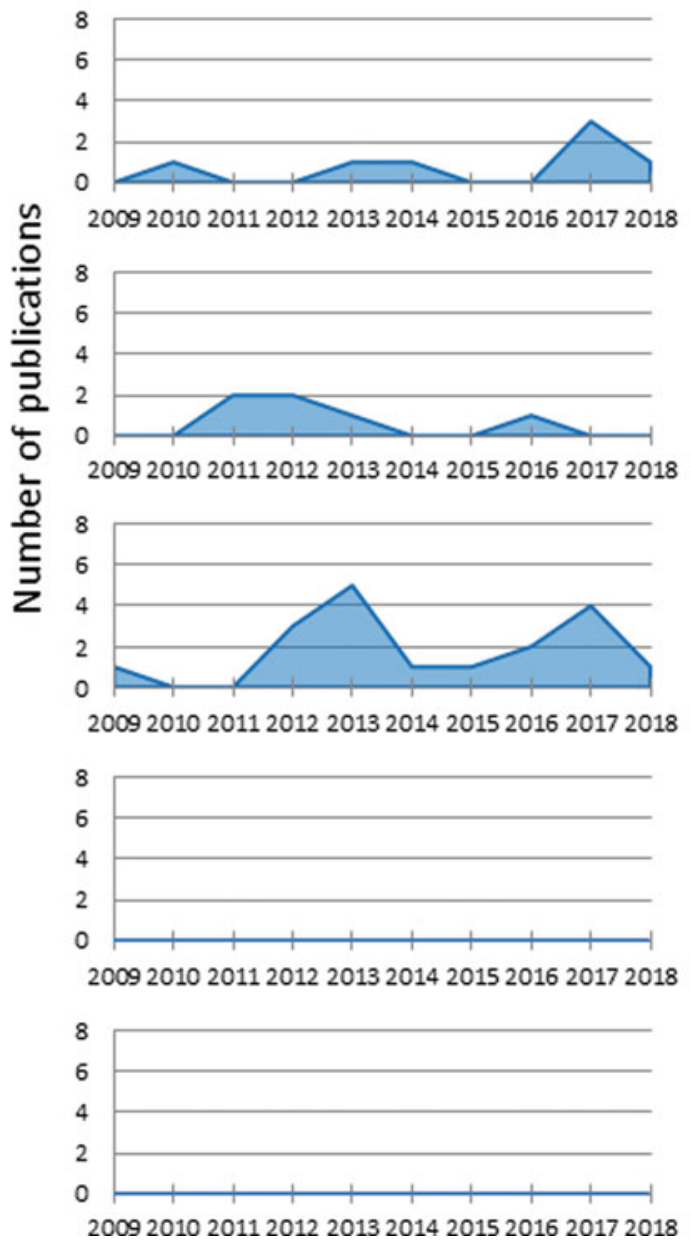

Mice
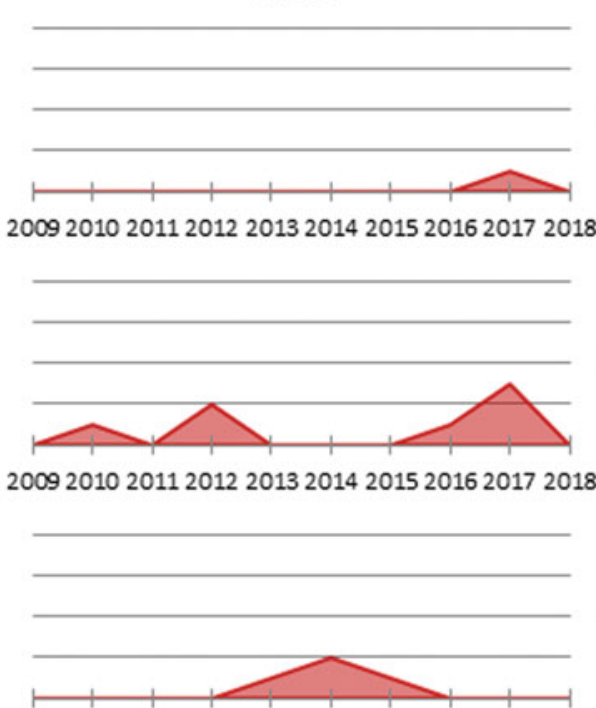

2009201020112012201320142015201620172018

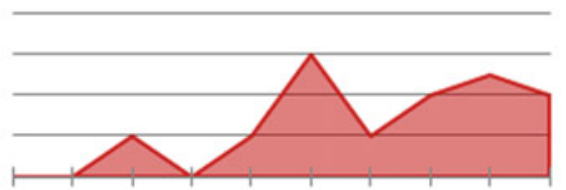

7.0T

2009201020112012201320142015201620172018

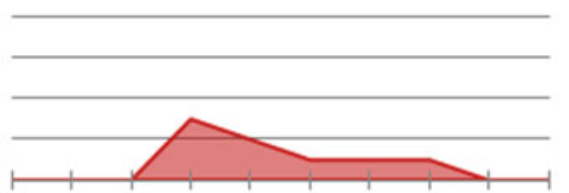

$9.4 \mathrm{~T}$

2009201020112012201320142015201620172018

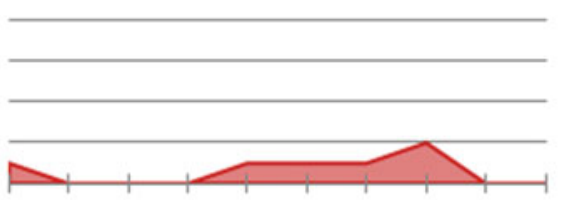

2009201020112012201320142015201620172018

\section{$11.7 \mathrm{~T}$}

$16.4 \mathrm{~T}$

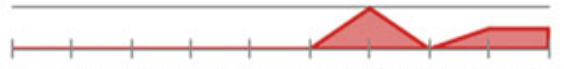

2009201020112012201320142015201620172018

Fig. 2 Number of selected publications ordered by magnetic field strength and year, within a total of 121 reports published in the past 10 years (2009-2018) for preclinical renal MRI in rats and mice 
The duty cycle defines the amount of time during which the gradient system can be run at maximum power amplitude (in \% time). This parameter depends not only on the gradient system specifications mentioned above but also on the specific MR imaging technique used. Typical duty cycles in existing preclinical scanners can reach almost $100 \%$. For this purpose, gradient coils are watercooled to ensure proper heat dissipation and temperature management.

The accuracy of the spatial representation depends on the quality of the linear magnetic field gradients and on the homogeneity of the static magnetic field (typically given in parts per million, $\mathrm{ppm}$ ). Any deviation will appear as object distortion in the image, and thus a correction procedure called "shimming" is typically performed. During system installation, the manufacturer places small metal plates inside the scanner bore to improve the uniformity of the main magnetic field in a static and permanent manner-this is "passive" shimming. The distribution of these ferromagnetic pieces is adjusted over several iterations until the achieved magnetic field uniformity meets the vendor's specification. In addition to passive shimming, resistive shim coils are used for $\mathrm{B}_{0}$ homogenization. These adjustments are performed with a shim calibration tool on an empty magnet, and therefore the $\mathrm{B}_{0}$ homogeneity becomes impaired as soon as a sample or subject (human or animal) is placed inside the magnet bore.

An "active" shimming procedure can be conducted during measurement adjustments with every MR scan. This is crucial for techniques such as fat suppression or MR spectroscopy, since the introduction of any matter-an object or animal-inevitably introduces local magnetic field perturbations of the main static magnetic field, which then must be actively compensated for. Especially at the boundaries between tissue and air-filled areas, where magnetic field distortions are pronounced due to the different magnetic susceptibility which can cause severe signal losses and image distortions. Corrective magnetic fields produced by electrical RF coils, designated as shim gradients [20] which are superimposed upon the main magnetic field. Typically, the shim gradient system contains a set of first-order gradients which impose linear field changes in the $\mathrm{X}-, \mathrm{Y}-$, and $\mathrm{Z}$-directions, and some second order gradients, which create spherical harmonics in the $\mathrm{Z}^{2}, \mathrm{ZX}, \mathrm{ZY} \mathrm{X}^{2}-\mathrm{Y}^{2}$, and $\mathrm{XY}$ directions [21]. Some MR systems are also equipped with more sophisticated shim gradients which provide third and fourth order correction terms [22].

Depending on the specific pulse sequence and imaging technique used, the scientist has to adjust these magnetic field corrections to meet certain quality prequisites. Especially with $\mathrm{T}_{2}{ }^{*}$-weighted sequences (e.g., gradient-echo or EPI sequences) the image quality improves with improved $B_{0}$ homogeneity. MR spectroscopic methods are even more demanding [23]. Usually, 
state-of-the-art MR systems provide automated methods to perform the $\mathrm{B}_{0}$ shimming task, but the user still needs to check the performance and correct the shim settings manually if necessary.

For $\mathrm{B}_{0}$ shimming a global approach covering the entire FOV, or a localized shim covering a target area can be used. In general, the smaller the region of interest the better the achievable $B_{0}$ homogeneity. In the case of renal MRI, the $\mathrm{B}_{0}$ homogeneity achieved after shimming across one kidney will be better than that for the whole abdomen, with air-filled lungs and gut. In the case of tissues that are moving due to respiration, cardiac activity or peristaltic motion, the magnetic field distortions change over time, which require different strategies. The time-averaged global shim would be the fastest and easiest solution, if this is sufficient. Alternatively, shimming-and also data acquisition-would be performed in synchrony with the breathing by using a trigger signal from a physiological monitor [24].

\section{Radio Frequency (RF) Coils}

Besides the static magnetic field and the spatial encoding with the gradient system, MRI requires transmission of RF pulses and reception of the MR signal from the sample or subject.

For signal excitation, the RF transmit resonator, or RF coil, sends RF pulses with a particular frequency, bandwidth, shape, duration, amplitude, and phase modulation to tilt the net magnetization vector oriented in parallel with the main magnetic field during equilibrium. $\mathrm{B}_{0}$ (typically assigned as the $\mathrm{Z}$-direction), is tilted into the perpendicular transverse $(\mathrm{X}-\mathrm{Y})$ plane by a given flip angle $\alpha$. After excitation, the magnetization precesses back to equilibrium and induces a signal in the RF receive coil (for a comprehensive review see [25]).

Depending on the transmission and reception capabilities, three general types of RF coils are commonly used: (1) transmitreceive $(\mathrm{Tx} / \mathrm{Rx})$ or transceiver $\mathrm{RF}$ coils, $(2)$ transmit-only $(\mathrm{Tx}) \mathrm{RF}$ coils, and (3) receive-only $(\mathrm{Rx}) \mathrm{RF}$ coils. These generally have two types of geometry: (1) volume RF coils and (2) surface RF coils. Both RF coil types and geometries determine the amount of tissue or organ coverage and sensitivity to the MR signal.

Volume RF resonators are designed to produce a uniform RF field over a large field-of-view (FOV), and are typically used as transmit-receive RF coils or transmit RF coils (using a surface RF coil for reception). The most widely used type of volume RF coils in both human and preclinical MRI are birdcage resonators [26, 27] driven in quadrature mode. This means that two spatially orthogonal RF channels are used for RF transmission as well as signal reception. This RF coil configuration provides a $41.4 \% \mathrm{SNR}$ gain over simple single channel or "linear" RF coils, due to the coherent 
signal increases which scale linearly with the number of channels, while the incoherent noise increases with the square root of the number of channels. For example, Chang et al. [28] used this RF coil setup in a renal hypoxia study in a mouse model after myocardial infarction of different sizes using BOLD MRI. Other types of volume resonators include saddle RF coils, Helmholtz RF coils, and the transverse electromagnetic (TEM) resonators, among others.

Smaller anatomical coverage and FOVs, but enhanced SNR, are provided by surface RF coils. Due to the principle of reciprocity, the closer the position of the RF coil is to the tissue of interest, the higher is the attainable SNR. However, the sensitivity drops with increasing distance to the RF coil surface, which thus results in a reduced FOV, and an inhomogeneous sensitivity profile [29]. Within a short distance of typically up to $\sim 1 \mathrm{~cm}$, a surface RF coil outperforms a volume RF coil in terms of sensitivity. Since the kidneys are located beneath the skin, surface RF coils positioned directly above are used for optimal signal reception in renal MRI. This is advantageous not only for X-nuclei MR imaging (see Subheading 5 ) but also for other applications with low SNR, such as perfusion or diffusion-weighted imaging.

Surface RF coils may be used as transmit/receive devices or as receive-only RF coils in conjunction with a volume RF coil used for homogeneous RF transmission. The first option is technically very simple since only one RF coil is necessary, which occupies less space. In the case of small animal imaging, it is most likely a simple singleturn loop with at least two tunable capacitances for tuning and matching. Images will show a strong spatially varying SNR decay, due to the addition of the inhomogeneous excitation and reception profiles [30]. This effect may be mitigated, for example, by using adiabatic excitation RF pulses [31].

The second option involves the use of two RF coils (Tx: volume RF coil, Rx: surface RF coil) which are tuned to the same resonant frequency. To avoid RF coupling between both coils, the surface RF coil must be detuned during transmission while the volume RF coil must be detuned during reception. Thus, both RF coils must include an option, using active and passive diodes, to allow for tuning and detuning during the MR experiment. This is the most common setup, since it improves the SNR in comparison with a $\mathrm{Tx} / \mathrm{Rx}$ volume $\mathrm{RF}$ coil, while still maintaining a homogeneous $\mathrm{B}_{1}$. For example, Hüper et al. [32] used a four-element quadrature surface receive RF coil in combination with a transmit volume RF coil to investigate renal perfusion in acute and chronic renal allograft rejection in translational mouse models. The use of the surface RF coil improved the SNR from 15 (with a standard transceive volume RF coil) to 35 .

Further SNR gains in small animal MRI are achieved with arrays of surface RF coils for signal reception [33], which are typically used together with a volume RF coil for signal excitation 
(see Notes 3 and 4). The use of RF coil arrays for brain imaging is especially widespread, and these are routinely offered by manufacturers. State-of-the-art small-animal MRI systems are usually equipped with 4-16 $\mathrm{Rx}$ channels, to which custom-tailored RF coils may be connected. Less popular are metamaterial-inspired RF coils, which have been developed for superficial tissue imaging in order to provide the best compromise of high SNR over a large FOV, while using only one receive channel [34].

Depending on the size of the sample and the RF coil, and their corresponding temperatures and resistances, their noise electronic contribution varies [35-37]. This factor is even more crucial in preclinical applications where the noise contribution of the RF coil, capacitors, sample, shield, receive electronics, and transmission lines are important [26]. This fact is exploited in small-animal imaging by the use of cryogenically cooled (cryo-cooled) RF coils. By lowering the temperature of the receiver chain, the noise contributions corresponding to temperatures and resistances can be reduced [38-40], thus achieving a significant increase in SNR, which has been reported in the literature (for a review see [15]). Unfortunately, only negligible effects are achieved in human MR $[15,41]$. With increasing size of the sample, the relative contribution to the noise increases, and eventually becomes the dominating noise source-this is the default situation for human MR. Thus, only small animal MR applications will benefit from this cooling approach, since the noise contributions from the sample and RF coil are in the same order of magnitude. The SNR gain achieved with such cryo-cooled RF coils is higher at lower static magnetic fields $[15,26,42]$. A recognized limitation of cryo-cooled surface RF coils is that only transceive capabilities are supported, resulting in nonuniform excitation and reception profiles, which might hamper quantitative measurements.

Another option for the small-animal MR imaging of the kidney is the use of clinical scanners together with RF coils tailored for the human hand/wrist or knee. These are typically 8 - to 16-element RF coil arrays which provide quasi-homogeneous excitation and reception profiles. Although these RF coils have a small size, they are not optimized for small-animal imaging and are not designed with matching/tuning capabilities. Moreover, RF coils tailored for rodent imaging are designed for a broad range of loadings (i.e., from almost empty to full loading, with a typical frequencydiameter product in the range $2-30 \mathrm{MHz} \cdot \mathrm{m}$ [26]), in comparison to the clinical setups. Therefore, these clinical RF coils will achieve a lower efficiency than that of dedicated RF coils designed for preclinical MR systems. Alternatively, RF coils tailored for renal MRI of small rodents provided by RF coil manufactures can be connected with clinical MR scanners to enhance the fill factor and SNR. 


\subsection{Commercially Available RF Coils Suitable for Kidney MRI in Rats}

In the following subsections, examples of possible RF coil setups are shown from different vendors. A list of the available MR system and RF coil vendors can be found in Table 2 .

Due to the considerable anatomical homology with humans, rats have become one of the most commonly use rodents in animal research, particularly, in preclinical MRI. With a weight of 150-450 g, both clinical and preclinical MR systems and RF coils can be used to perform renal MR experiments:

Clinical MR System

- Tx/Rx wrist RF coil (e.g., Siemens eight-channel wrist RF coil, Fig. 3A).

- Tx/Rx knee RF coil (e.g., Siemens 15-channel knee RF coil, Fig. 3B).

Preclinical MR System

- $\mathrm{Tx} / \mathrm{Rx}$ volume resonator (e.g., Bruker Biospin, Ettlingen, Germany, quadrature rat body $\mathrm{RF}$ coil with inner diameter (ID) $=72 \mathrm{~mm}$ (Fig. 3C), $82 \mathrm{~mm}$ or $86 \mathrm{~mm}$; RAPID Biomed, Rimpar, Germany $2 \times 4$-channel volumetric rat array with $\mathrm{ID}=72 \mathrm{~mm})$.

- $\mathrm{Tx} / \mathrm{Rx}$ quadrature volume resonator (e.g., MRI.TOOLS GmbH, Berlin, Germany, supporting an ID ranging from $54 \mathrm{~mm}$ to $86 \mathrm{~mm}$ and magnetic field strengths of $3.0 \mathrm{~T}, 4.7 \mathrm{~T}$, 7.0 T, 9.4 T, and 11.T (Fig. 3D).

- Rx surface RF coil (e.g., 2 × 2 RAPID Biomed, Rimpar, Germany) rat cardiac surface array RF coil; $4 \times 1$ element (e.g., Bruker Biospin, Ettlingen, Germany) rat cardiac surface array RF coil, Fig. 3E) in combination with $\mathrm{Tx}$ volume resonator (e.g., Bruker rat body RF coils with ID $72 \mathrm{~mm}$ (Fig. 3F), $82 \mathrm{~mm}$ or $86 \mathrm{~mm})$.

\subsection{Commercially Available RF Coils Suitable for Kidney MRI in Mice}

Mice are the most commonly used rodent in biomedical research. Their genetic similarity to humans has made them extremely useful, allowing the development of mouse models of many human diseases. Mice have an average weight of 15-40 g, making them too small for standard human RF coils:

\section{Clinical System}

- There are no standard human RF coils that are small enough to be suitable for mice MRI. Alternatively, a $\mathrm{Tx} / \mathrm{Rx}$ quadrature volume resonator (MRI.TOOLS GmbH, Berlin, Germany), supporting an ID ranging from $30 \mathrm{~mm}$ to $45 \mathrm{~mm}$ and magnetic field strengths of 3.0 and 7.0 T can be used for renal MRI with clinical MR scanners (Fig. 4A). 


\section{Table 2}

List of vendors of MR equipment and RF coils

\begin{tabular}{lll}
\hline & Vendor & Website \\
\hline Clinical MRI & Canon Medical Systems & us.medical.canon \\
\hline General Electric Medical Systems Inc. & www.gehealthcare.com \\
\hline MRI Devices Corp. & www.allmri.com \\
\hline MR Instruments Inc. & www.mrinstruments.com \\
\hline MRI.TOOLS GmbH & www.mritools.de \\
\hline NOVA Medical Inc. & www.novamedical.com \\
\hline RAPID Biomedical GmbH & www.rapidbiomed.de \\
\hline Philips Medical Systems & www.medical.philips.com \\
\hline ScanMed & www.scanmed.com \\
\hline Siemens Medical Systems & www.siemens-healthineers.com \\
\hline Tesla Engineering Limited & Bruker BioSpin MRI GmbH & www.bruker.com \\
\hline Preclinical MRI & Doty Scientific, Inc. & www.dotynmr.com \\
\hline MR Solutions & www.mrsolutions.com \\
\hline MRI.TOOLS GmbH & www.mritools.de \\
\hline RAPID Biomedical GmbH & www.rapidbiomed.de \\
\hline
\end{tabular}

Preclinical System

- $\mathrm{Tx} / \mathrm{Rx}$ volume resonator (Bruker Biospin, Ettlingen, Germany) quadrature mouse body RF coil with $\mathrm{ID}=35 \mathrm{~mm}$, Fig. $4 \mathrm{~B}$ ); $2 \times 4$-channel volumetric mouse array (RAPID Biomed, Rimpar, Germany) with $35 \mathrm{~mm}$ ID.

- Tx/Rx quadrature volume resonator (MRI.TOOLS GmbH, Berlin, Germany, supporting an ID ranging from $30 \mathrm{~mm}$ to $45 \mathrm{~mm}$ and magnetic field strengths of $3.0 \mathrm{~T}, 4.7 \mathrm{~T}, 7.0 \mathrm{~T}$, 9.4 T, and 11.T (Fig. 4A).

- Rx surface RF coil (e.g., RAPID Biomed, Rimpar, Germany) $2 \times 2$ mouse cardiac surface array RF coil; $2 \times 2$ element mouse cardiac surface array RF coil (e.g., Bruker Biospin, Ettlingen, Germany, Fig. 4B) in combination with Tx volume resonator (e.g., Bruker Biospin, Ettlingen, Germany) quadrature mouse body RF coils with ID $72 \mathrm{~mm}$ (as shown in Fig. 4E), $82 \mathrm{~mm}$ or $86 \mathrm{~mm})$.

- Tx/Rx $2 \times 2$ element array rat/mouse CryoProbe (e.g., Bruker Biospin, Ettlingen, Germany, Fig. 4C). 

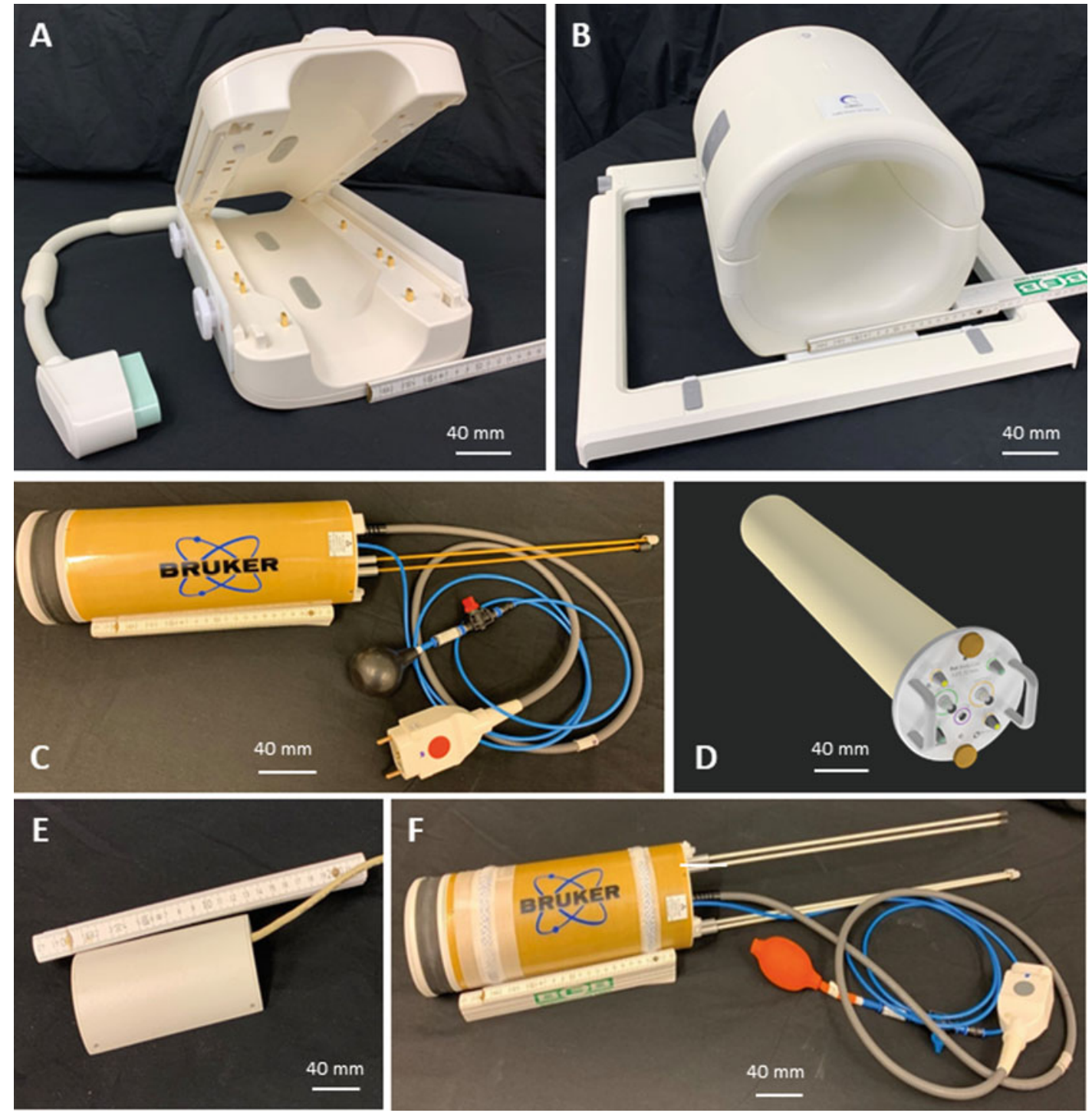

Fig. 3 Example RF coils suitable for kidney MRI of the rat. (a) Siemens 8-channel wrist RF coil, (b) Siemens 15-channel knee RF coil, (c) Bruker quadrature rat body RF coil with inner diameter (ID) $72 \mathrm{~mm}$, (d) MRI.TO0LS $\mathrm{GmbH}$ quadrature rat body RF coil with ID of $72 \mathrm{~mm},(\mathbf{e}) 4 \times 1$ element Bruker rat cardiac Rx surface array RF coil, (f) Bruker rat body Tx volume resonator with ID $72 \mathrm{~mm}$.

\section{$5 \quad$ Multinuclear Imaging}

The most frequent MRI method in biomedical research is proton

$\left({ }^{1} \mathrm{H}\right)$ imaging. Hydrogen is the most abundant nucleus in living organisms. It also is the nucleus which is the most sensitive to interactions with an external magnetic field, due to its high gyromagnetic ratio $(\gamma=42.576 \mathrm{MHz} / \mathrm{T})$. 

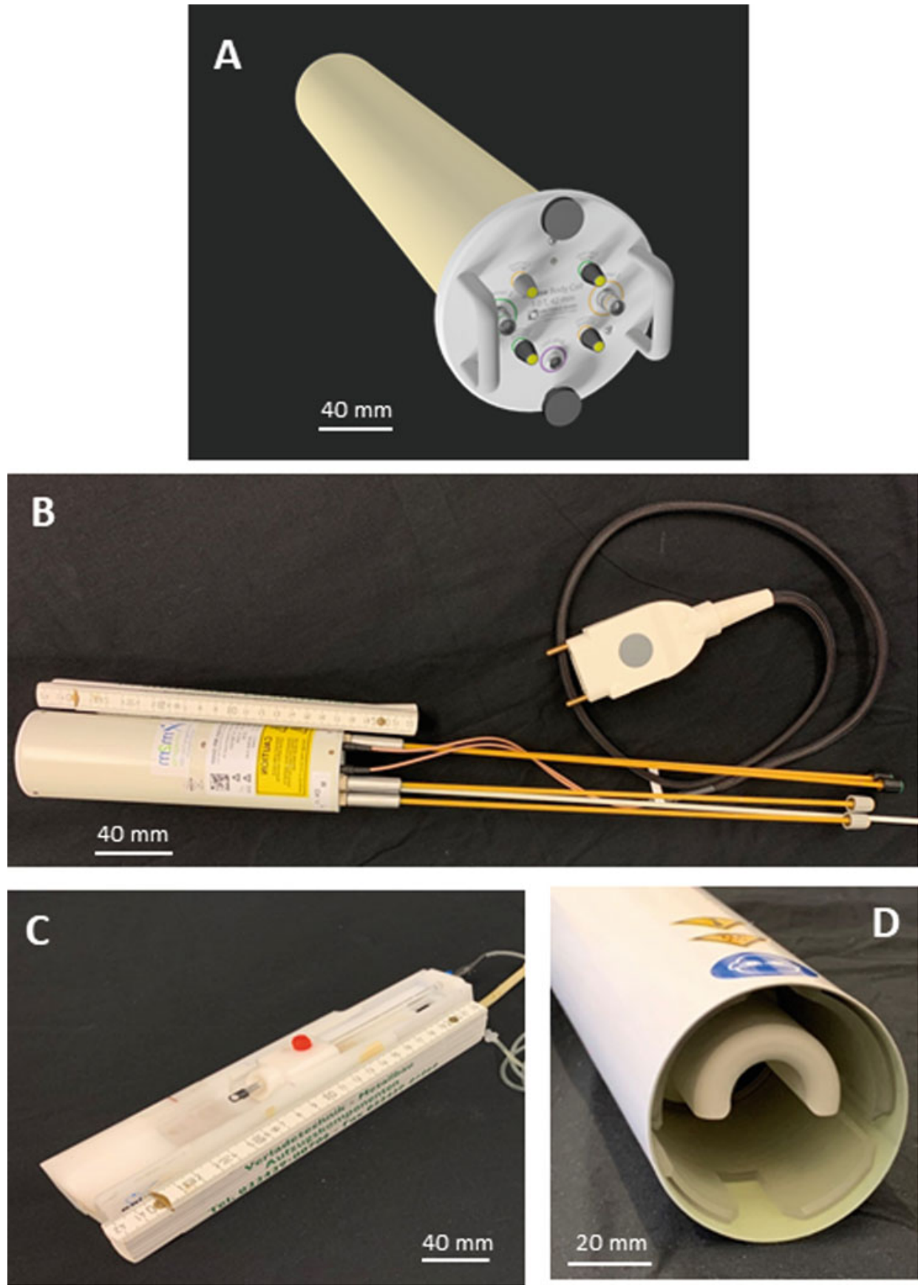

Fig. 4 Example RF coils suitable for kidney MRI of the mouse. (a) MRI.TOOLS GmbH quadrature mouse body RF coil with an ID = $42 \mathrm{~mm}$, (b) Bruker quadrature mouse body RF coil with $35 \mathrm{~mm} \mathrm{ID}$, (c) Bruker $2 \times 2$ element mouse cardiac surface array RF coil, (d) Tx/Rx quadrature surface RF coil with cryo-cooling technology (CryoProbe) 
In preclinical renal MRI, structural and functional information is typically investigated using in vivo ${ }^{1} \mathrm{H}$ MR imaging of the kidney. Techniques such as $\mathrm{T}_{2} / \mathrm{T}_{2}{ }^{*}$ mapping, arterial spin labeling (ASL), dynamic contrast-enhanced imaging (DCE-MRI) or diffusionweighted imaging (DWI) are performed in order to study the oxygenation, perfusion, clearance rate, and water and metabolite motion within the kidneys.

Physiometabolic probing is supported by imaging and spectroscopic applications of other nuclei such as ${ }^{13} \mathrm{C},{ }^{17} \mathrm{O},{ }^{23} \mathrm{Na},{ }^{35} \mathrm{Cl},{ }^{39} \mathrm{~K}$, ${ }^{31} \mathrm{P}$, and ${ }^{19} \mathrm{~F}$ [43]. The detection of $\mathrm{X}$-nuclei is challening because of the low intrinsic SNR due to the smaller $\gamma$, compared to ${ }^{1} \mathrm{H}$, and by the lower abundance of these nuclei in biological tissues.

Increasing $B_{0}$ is the most effective way to increase sensitivitythe benefits of higher $\mathrm{B}_{0}$ are unique metabolic and functional information, as well as outstanding anatomical detail [4447]. X-nuclei MR also benefits from the increase in SNR achieved with cryo-cooled transceive surface RF coils, which are currently available for ${ }^{13} \mathrm{C},{ }^{31} \mathrm{P},{ }^{19} \mathrm{~F}$, and ${ }^{1} \mathrm{H}[48,49]$.

Changes in the biodistribution of endogenous metabolites that can be detected with ${ }^{23} \mathrm{Na}$ - or ${ }^{31} \mathrm{P}$-MR have been demonstrated to be markers of renal disease [50-53]. For more details on preclinical ${ }^{23} \mathrm{Na}-\mathrm{MRI}$ protocols, see the chapters by Grist JT et al. "Sodium $\left({ }^{23} \mathrm{Na}\right) \mathrm{MRI}$ of the Kidney: Basic Concept" and "Sodium $\left({ }^{23} \mathrm{Na}\right)$ MRI of the Kidney: Experimental Protocol." Similarly, renal perfusion, oxygenation, and inflammatory cell infiltration can be investigated using ${ }^{19}$ F-MRI [54-57]. See the chapters by Grist JT et al. "Sodium $\left({ }^{23} \mathrm{Na}\right) \mathrm{MRI}$ of the Kidney: Basic Concept" and "Sodium $\left({ }^{23} \mathrm{Na}\right)$ MRI of the Kidney: Experimental Protocol" on how to perform ${ }^{19} \mathrm{~F}$-MRI renal studies in rodents.

Hyperpolarization techniques have great potential for explorations into renal metabolic diseases (e.g., hyperpolarized ${ }^{13} \mathrm{C}-\mathrm{MRI}$ ), where SNR is boosted up to a 20,000-fold compared to conventional ${ }^{13} \mathrm{C}-\mathrm{MRI}$ [58]. Experimental protocols to perform hyperpolarized ${ }^{13} \mathrm{C}$-MRI in preclinical studies are provided in the chapters by von Morze $\mathrm{C}$ et al. "Hyperpolarized Carbon $\left({ }^{13} \mathrm{C}\right) \mathrm{MRI}$ of the Kidneys: Basic Concept" and by Laustsen C et al. "Hyperpolarized Carbon $\left({ }^{13} \mathrm{C}\right)$ MRI of the Kidney: Experimental Protocol."

\section{Physiological Monitoring}

A major difference between clinical and preclinical MRI is the need to anesthetize the animals prior to imaging. Since most anaesthetic drugs produce physiological changes (temperature decrease, depression of cardiorespiratory function, etc.), monitoring the anaesthetized animals is a very crucial aspect in preclinical MRI studies. Even in the most simple case, that is, the scanning of spontaneously breathing animals without any further intervention 
or treatment, respiration and temperature of the animal has to be monitored continuously during the scan in order to guarantee the appropriate anaesthetic status and to avoid hypothermia. In particular, hypothermia is a critical issues in rodent imaging, since mice have a low mass vs. a large surface which promotes heat loss $[59,60]$. This problem is less severe in rats due to their larger weight, but it is not negligible [61]. Heating systems must be therefore used to maintain temperature homeostasis.

Physiological monitoring of the animal is even more essential in functional MR imaging, since changes may produce confounding effects on the outcome of the MR experiment [62]. Careful monitoring and recording of physiological data should be performed at all times during the experiment to ensure the validity of the results and their reproducibility. The frequency of physiological monitoring depends on the invasiveness of the experimental procedure as well as the time after induction of anaesthesia. As a rule of thumb, the more invasive the procedure and the longer the time under anaesthesia, the more frequent the physiological monitoring of the animal should be performed. Additionally, the correct positioning of the animals on the animal bed systems, along with careful intubation (if needed) are essential to ensure respiratory and circulatory comfort during imaging, and to promote recovery of the animal after the experiment.

Typical physiological parameters monitored during MR experiments include temperature, heart rate, and respiration rate. Many manufacturers already offer these three monitoring channels, and are ready to use these signals for the purpose of gating or triggering the MR imaging techniques. Additionally, information on blood pressure, blood oxygen saturation, transcutaneous $\mathrm{pCO}_{2}$, or $\mathrm{pH}$ can also be collected. In some cases, invasive withdrawal of blood samples is necessary-this is a very difficult task since the sampling has to be executed using long tubes with considerable dead volumes and very limited visual control.

Finally, all equipment must allow for remote physiological monitoring, and strictly comply with MR safety requirements. Several integrated animal handling solutions for physiological monitoring and anaesthesia are commercially available. Examples are given in Table 3 .

\section{Practical Points to Consider When Planning a Study}

The aim of this chapter is to outline the main hardware components required for preclinical MRI of the kidney and to guide the appropriate hardware selection to meet the individual needs.

This section is intended to provide a summary of the prequisites needed and to help prepare for preclinical renal MR applications: 
Table 3

Examples of commercially available equipment for physiological monitoring in preclinical MRI

\begin{tabular}{|c|c|}
\hline $\begin{array}{l}\text { Physiological parameter } \\
\text { (s) }\end{array}$ & Examples of vendor equipment \\
\hline Temperature & $\begin{array}{l}\text { Omniflex (Neoptix, Inc., Canada) } \\
\text { Fiber optic temperature probes (SA Instruments, Inc. Stony Brook, NY, } \\
\quad \text { USA) } \\
\text { RightTemp (Kent Scientific Corp., Torrington, CT, USA) } \\
\text { MultiSens (Opsens Solutions, Canada) }\end{array}$ \\
\hline $\begin{array}{l}\text { Respiration rate/ECG } \\
\text { and gating }\end{array}$ & $\begin{array}{l}\text { BioVet }(\mathrm{m} 2 \mathrm{~m} \text { imaging, Cleveland, OH, USA) } \\
\text { Model } 1030 \text { Monitoring \& Gating system (Small Animall Instruments, Inc. } \\
\text { Stony Brook, NY, USA) }\end{array}$ \\
\hline Blood pressure & CODA monitor (Kent Scientific Corp., Torrington, CT, USA) \\
\hline $\begin{array}{l}\text { Blood oxygen saturation, } \\
\text { heart rate }\end{array}$ & $\begin{array}{l}\text { Pulse oximeter (SA Instruments, Inc. Stony Brook, NY, USA) } \\
\text { MouseOx (STARR Life Sciences Corp., USA) }\end{array}$ \\
\hline Transcutateous $\mathrm{pCO}_{2}$ & $\begin{array}{l}\text { Capnograph (SA Instruments, Inc. Stony Brook, NY, USA) } \\
\text { CapnoScan (Kent Scientific Corp., Torrington, CT, USA) }\end{array}$ \\
\hline Several & $\begin{array}{l}\text { PhysioSuite (Kent Scientific Corp., Torrington, CT, USA) } \\
\text { PLUGSYS (Harvard Apparatus, Holliston, MA, USA) }\end{array}$ \\
\hline
\end{tabular}

\subsection{MRI System}

\subsubsection{Clinical or}

Preclinical?

\subsubsection{Should I Consider} Moving to a High Magnetic Field Strength?
With a 3.0 T clinical MR system, renal MRI of rats is feasible with acceptable image quality and spatial resolution. However, if possible, we recommend to use a dedicated ultrahigh field MR system tailored for small-animal imaging with a magnetic field strength of 7.0 or $9.4 \mathrm{~T}$. The reason is that the SNR gain achieved with preclinical MR scanners compared to that at lower $\mathrm{B}_{0}$ is instrumental for achieving good spatial and temporal resolution, as well as for reducing the scan times needed to perform MR examinations.

As discussed above (see Subheading 2), the use of a higher magnetic field strength results in an increase in $S N R$ proportional to $B_{0}$ $\left(\mathrm{SNR} \propto \mathrm{B}_{0}^{1.65}\right)$. This means that renal $\mathrm{MR}$ images will achieve greater SNR that can be traded off for to achieve higher spatial resolution or reduced scan time as needed (see Table 1). Also, higher $\mathrm{B}_{0}$ increases the BOLD effect. In general, all methods based on $\mathrm{T}_{2}{ }^{*}$-weighting will benefit from an increase in the magnetic field strength. MR spectroscopy applications also profit from the increased $\mathrm{B}_{0}$. Therefore, we strongly recommend the use of a high $\mathrm{B}_{0}$ for these MR applications. However, not all facilities have such high field MR scanners readily available, in which case it might be advisable to perform the experiments using a lower magnetic field system, as opposed to moving animals, equipment, and personnel to another facility. 
7.1.3 Higher Magnetic Fields Are All Rainbows and Unicorns, and Should Always Be Used, Right?

7.1.4 OK, But ... Which $B_{0}$ Is Everyone Else Using?

7.1.5 What About the Gradient System?

\subsection{RF Coil Setup}

7.2.1 Which Kind of RF Coil Should I Use to Get the Most Juice from My Clinical MR Scanner?
Not necessarily! Be aware that higher magnetic field strengths suffer from greater $B_{0}$ and $B_{1}$ inhomogeneities than lower field strengths, which must be corrected in order to fully benefit from the boost in $\mathrm{SNR}$. Also, due to the $\mathrm{B}_{0}$-dependent changes of the relaxation properties, the method-specific and protocol-specific imaging parameters have to be adopted in order to generate similar image contrasts that are comparable to those achieved at lower $\mathrm{B}_{0}$. If the focus is the transfer of MR methods from preclinical models to clinical applications, use of the same static magnetic field strength is advisable. The "loss" of SNR may be partially compensated for by the use of cryo-cooled RF coils, which would be more cost-effective than the procurement of a high field small animal MR scanner.

The move to higher magnetic field strengths is supported by a large body of literature (see Figs. 1 and 2) which reports the most typical magnetic field strengths for rats to be 3.0 and $9.4 \mathrm{~T}$ and for mice to be 7.0 and $9.4 \mathrm{~T}$. The continued use of $3.0 \mathrm{~T}$ scanners for rats is mainly due to the use of readily available clinical MR systems, which are then adapted for rat MR experiments. However, these are typically performed using hand/wrist or knee RF coils tailored for clinical applications in humans, and are not optimized for animal imaging, with the main disadvantages being a loss of signal due to incorrect loading, and the inability to match and tune the RF coil.

Depending on the MR application of your choice, a high gradient slew rate might be needed. Fast imaging techniques need fast gradient switching speeds, since this parameter influences the minimum achievable repetition time (TR), echo time (TE), and the echo spacing. However, a higher slew rate means a greater monetary investment will be needed at the acquisition of a new MR scanner. Equally, the duty cycle is a crucial parameter in hardwaredemanding sequences, which generally includes all fast imaging techniques. We recommend the procurement of a gradient system tailored according to the individual experimental needs.

The best option is to use a hand/wrist RF coil; a knee RF coil is a second-best option. Both of these are RF coil arrays which provide high SNR and full FOV coverage. However, these RF coils are tailored for clinical applications in humans and are not optimized for animal imaging, with the main disadvantages being a loss of signal due to incorrect loading and the inability to match and tune the RF coil. Also, the clinical MR system must be adapted for rat MR experiments (mice are too small and do not provide enough loading in clinical systems). Alternatively, dedicated RF coils provided by RF coil vendors which are customized for renal MRI of rats can be connected to the clinical MR scanner. 
7.2.2 Which RF Coil Setups Are the Most Suitable for Renal Applications in My Preclinical MR Scanner?

\section{$7.3 \quad X$-Nuclei}

\subsubsection{What If I Want} to Perform X-Nuclei MRI/MRS?

\subsection{Animal Handling and Physiological Monitoring}

\author{
7.4.1 How Should \\ I Position the Rodent \\ in the Animal Bed Prior \\ to the Experiment?
}

\subsubsection{What Is} the Essential Physiological Monitoring that Should Be Performed During Small-Animal Renal MR Experiments?

\subsubsection{What Other} Physiological Parameters Can I Measure, and Which Equipment Should I Use?
The anatomical position of the kidneys suggests placement of the RF coil in close proximity to the dorsal skin of the animal. We recommend the use of a surface RF coil for signal reception together with a volume RF coil for excitation. This is the most common setup since it improves the SNR in comparison with a $\mathrm{Tx} / \mathrm{Rx}$ volume $\mathrm{RF}$ coil, while still maintaining a homogeneous $\mathrm{B}_{1}$. Also, be aware that this setup needs the synchronized detuning of the RF coils in turns in order to avoid RF coupling between the two coils (the surface RF coil must be detuned during transmission while the volume RF coil must be detuned during reception).

Broadband RF chains, including the transmission path and the receive path, together with $\mathrm{RF}$ coils specifically tailored for $\mathrm{X}$-nuclei are needed. Due to the typically low SNR inherent to $\mathrm{X}$-nuclei MR applications, a good option would be the use of a cryo-cooled RF coil. Imaging of a single kidney can be performed in mice using a mouse cryo-cooled RF coil. To image both kidneys simultaneously in mice, a rat cryo-cooled RF coil could be used. However, bear in mind that cryo-cooled RF coils are designed as transceive surface RF antennae, and provide inhomogeneous transmit and sensitivity profiles. Therefore, if your MR application needs exact $T_{1}$ contrasts or quantification based on absolute signal intensities, a $\mathrm{B}_{1}$ correction must be performed.

Please also see the chapter by Kaucsar T et al. "Preparation and Monitoring of Small Animals in Renal MRI."

This depends on the kind of RF coil being used. Typically, the kidneys are measured from the dorsal side using a combination of a volume RF coil for RF pulse transmission and a surface RF coil to receive the $\mathrm{MR}$ signal. The volume $\mathrm{RF}$ coil will be placed inside the bore and fixed, while the surface RF coil is typically displaceable or is fixed on the animal bed. The position of the animal will then be adapted according to this setup.

The essential physiological monitoring includes respiration, which is also used for triggering, and body temperature, which should be maintained at a normal level.

A wide variety of MR compatible equipment exists in the market that allows for measurement of temperature, respiration rate, blood pressure, blood oxygen saturation and respiration rate, and transcutaneous $\mathrm{pCO}_{2}$, among others. See Table 3 for exemples of commercially available equipment for physiological monitoring in preclinical MRI. 


\subsection{Image \\ Acquisition}

7.5.1 Should I Perform $B_{0}$ Shimming in Every MR Study?

7.5.2 Should I Always Do Triggering of the Signal? What Are my Options?
Preferably, yes. Active shimming protocols homogenize the $\mathrm{B}_{0}$ field in a small region of interest, and help to improve the performance of $\mathrm{T}_{2}{ }^{*}$-weighted MR protocols such as EPI. For performing MRS of the kidney (or any other organ), shimming should always be completed during the MR study adjustments. In general, we recommend to always perform shimming in every renal MR study.

Renal MR imaging should be synchronized with animal respiration, preferably triggered to the exhalation phase $[32,63]$. If possible, the animals should be allowed to breathe spontaneously under anaesthesia.

\section{Notes}

1. After the decade of the brain and the (re)discovery of the brain-heart-kidney network, a new era of kidney imaging is expected. The most important brain research tool is fMRI, and it has served as strong motivation for continued technological improvements in hardware development. Similar techniques are valuable for renal imaging. BOLD-based and $\mathrm{BV} / \mathrm{BF}-$ based techniques greatly benefit from higher magnetic fields, providing a higher SNR, a higher CNR, and better spatial resolution. Nevertheless, the use of higher magnetic fields brings new problems that call for new hardware solutions.

2. Although there is an increased demand on gradient performance, this will exacerbate the problems associated with acoustic noise. New gradient designs should take into account the acoustic noise pressure level as a parameter. Gradient systems that are targeted to the region of interest only may be a solution. The higher gradient performance with increased bandwidth, as well as the increased physiological noise, reduce the gain in the SNR. A limit of the tissue contribution to thermal noise may be found by new RF coil designs.

3. Modern MRI systems come equipped with multiple receiver units allowing parallel acquisition. A combination of small, multielement receive-only RF coils with parallel imaging is a game changer in the way MRI data are acquired. Reducing the RF coil size will allow for the acquisition of data from a small region of interest with higher sensitivity, because of the close proximity of the RF coil to the region, and thus limited noise contribution from tissue. The use of multiple elements, which are decoupled from each other, will help to achieve extended tissue coverage.

4. A problem associated with parallel imaging arises in the storage and processing of large amounts of data. Therefore, considerable effort must be invested into the handling of "Big Data." Boosting the data transfer rate, the use of array processors and the application of renal MRI will push the limits of data science. 
Acknowledgments

This work was funded in part (Thoralf Niendorf, Andreas Pohlmann) by the German Research Foundation (Gefoerdert durch die Deutsche Forschungsgemeinschaft (DFG), Projektnummer 394046635, SFB 1365, RENOPROTECTION. Funded by the Deutsche Forschungsgemeinschaft (DFG, German Research Foundation), Project number 394046635, SFB 1365, RENOPROTECTION).

This publication is based upon work from COST Action PARENCHIMA, supported by European Cooperation in Science and Technology (COST). COST (www.cost.eu) is a funding agency for research and innovation networks. COST Actions help connect research initiatives across Europe and enable scientists to enrich their ideas by sharing them with their peers. This boosts their research, career, and innovation.

PARENCHIMA (renalmri.org) is a community-driven Action in the COST program of the European Union, which unites more than 200 experts in renal MRI from 30 countries with the aim to improve the reproducibility and standardization of renal MRI biomarkers.

\section{References}

1. de Graaf RA, Brown PB, McIntyre S, Nixon TW, Behar KL, Rothman DL (2006) High magnetic field water and metabolite proton $\mathrm{T}_{1}$ and $\mathrm{T}_{2}$ relaxation in rat brain in vivo. Magn Reson Med 56(2):386-394. https:// doi.org/10.1002/mrm.20946

2. Ladd ME (2018) The quest for higher sensitivity in MRI through higher magnetic fields. Z Med Phys 28(1):1-3. https://doi.org/10. 1016/j.zemedi.2017.12.001

3. Moser E, Laistler E, Schmitt F, Kontaxis G (2017) Ultra-high field NMR and MRI-the role ofmagnet technology to iIncrease sensitivity and specificity. Front Phys 5:33. https:// doi.org/10.3389/fphy.2017.00033

4. Niendorf T, Barth M, Kober F, Trattnig S (2016) From ultrahigh to extreme field magnetic resonance: where physics, biology and medicine meet. MAGMA 29(3):309-311. https://doi.org/10.1007/s10334-016-05641

5. Hoult DI (2000) Sensitivity and power deposition in a high-field imaging experiment. J Magn Reson Imaging 12:46-67. https://doi. org/10.1002/1522-2586(200007)

12:1<46::AID-JMRI6>3.0.CO;2-D

6. Hoult DI, Richards RE (1976) The signal-tonoise ratio of the nuclear magnetic resonance experiment. J Magn Reson 24(1):71-85. https://doi.org/10.1016/0022-2364(76) 90233-X

7. Pohmann R, Speck O, Scheffler K (2016) Signal-to-noise ratio and MR tissue parameters in human brain imaging at 3,7, and 9.4 Tesla using current receive coil array. Magn Reson Med 75:801-809. https://doi.org/10.1002/ mrm. 25677

8. Guérin B, Villena JF, Polimeridis AG, Adalsteinsson E, Daniel L, White JK, Wald LL (1980) The ultimate signal-to-noise ratio in realistic body models. Magn Reson Med 78:1969-1980. https://doi.org/10.1002/ mrm.26564

9. Merkle EM, Dale BM (2005) Abdominal MRI at $3.0 \mathrm{~T}$ : the basics revisited. Am J Roentgenol 186(6):1524-1532. https://doi.org/10. 2214/AJR.05.0932

10. Rooney WD, Johnson G, Li X, Cohen ER, Kim SG, Ugurbil K, Springer CS (2007) Magnetic field and tissue dependencies of human brain longitudinal ${ }^{1} \mathrm{H}_{2} \mathrm{O}$ relaxation in vivo. Magn Reson Med 57(2):308-318. https://doi.org/ $10.1002 / \mathrm{mrm} .21122$

11. Seehafer JU, Kalthoff D, Farr TD, Wiedermann D, Hoehm M (2010) No increase of the blood oxygenation level-dependent functional magnetic resonance imaging signal with higher field strength: implications for 
brain activation studies. J Neurosci 30 (15):5234-5241. https://doi.org/10.1523/ JNEUROSCI.0844-10.2010

12. Schenck JF (1995) Imaging of brain iron by magnetic resonance: $T_{2}$ relaxation at different field strengths. J Neurol Sci 134:10-18. https://doi.org/10.1016/0022-510X(95) 00203-E

13. Duyn JH (2012) The future of ultra-high field MRI and fMRI for study of the human brain. NeuroImage 62:1241-1248. https://doi.org/ 10.1016/j.neuroimage.2011.10.065

14. Baldanchani P, Naidich TP (2015) Ultra-highfield MR neuroimaging. Am J Neuroradiol 36 (7):1204-1215. https://doi.org/10.3174/ ajnr.A4180

15. Niendorf T, Pohlmann A, Reimann HM, Waiczies H, Peper E, Huelnhagen T, Seeliger E, Schreiber A, Kettritz R, Strobel K, Ku MC, Waiczies S (2015) Advancing cardiovascular, neurovascular, and renal magnetic resonance imaging in small rodents using cryogenic radiofrequency coil technology. Front Pharmacol 6:255. https://doi.org/10. 3389 /fphar.2015.00255

16. Schick F (2005) Whole-body MRI at high field: technical limits and clinical potential. Eur Radiol 15(5):946-959. https://doi.org/ $10.1007 / \mathrm{s} 00330-005-2678-0$

17. Van de Moortele PF, Akgun C, Adriany G, Moeller S, Ritter J, Collins CM, Smith MB, Vaughan JT, Ugurbil K (2005) $B_{1}$ destructive interferences and spatial phase patterns at $7 \mathrm{~T}$ with a head transceiver array RF coil. Magn Reson Med 54:1503-1518. https://doi.org/ $10.1002 / \mathrm{mrm} .20708$

18. Niendorf T, Graessl A, Thalhammer C, Dieringer MA, Kraus $\mathrm{O}$, Santoro D, Fuchs $\mathrm{K}$, Hezel F, Waiczies S, Ittermann B, Winter L (2013) Progress and promises of human cardiac magnetic resonance at ultrahigh fields: a physics perspective. J Magn Reson 229:208-222. https://doi.org/10.1016/j. jmr.2012.11.015

19. Fiedler T, Ladd ME, Bitz AK (2017) SAR simulations \& safety. NeuroImage 168:33-58. https://doi.org/10.1016/j.neuroimage. 2017.03.035

20. Stockmann JP, Wald LL (2018) In vivo $B_{0}$ field shimming methods for MRI at 7T. NeuroImage 168:71-87. https://doi.org/10.1016/ j.neuroimage.2017.06.013

21. Juchem C, de Graaf RA (2016) $B_{0}$ magnetic field homogeneity and shimming for in vivo magnetic resonance spectroscopy. Anal Biochem 529:17-29. https://doi.org/10.1016/ j.ab.2016.06.003
22. Pan JW, Lo KM, Hetherington HP (2012) Role of very high order and degree $\mathrm{B}_{0}$ shimming for spectroscopic imaging of the human brain at 7 Tesla. Magn Reson Med 68 (4):1007-1017. https://doi.org/10.1002/ mrm.24122

23. Motyka S, Moser P, Hingerl L, Hangel G, Heckova E, Strasser B, Eckstein K, Robinson SD, Poser BA, Gruber S, Trattnig S, Bogner W (2019) The influence of spatial resolution on the spectral quality and quantification accuracy of whole-brain MRSI at $1.5 \mathrm{~T}, 3 \mathrm{~T}, 7 \mathrm{~T}$, and 9.4T. Magn Reson Med 82(2):551-565. https://doi.org/10.1002/mrm.27746

24. Meyer A, Stolz K, Dreher W, Bergemann J, Thimmashetty VH, Lueschen N, Azizi Z, Khobragade V, Maedler K, Kuestermann E (2015) Manganese-mediated MRI signals correlate with functional $\beta$-cell mass during diabetes progression. Diabetes 64(6):2138-2147. https://doi.org/10.2337/dbl4-0864

25. Gruber B, Froeling M, Leiner T, Klomp DWJ (2018) RF coils: a practical guide for nonphysicists. J Magn Reson Imaging 48:590-604. https://doi.org/10.1002/jmri.26187

26. Doty FD, Entzminger G, Kulkarni J, Pamarthy K, Staab JP (2007) Radio frequency coil technology for small-animal MRI. NMR Biomed 20:304-325. https://doi.org/10. $1002 / \mathrm{nbm} .1149$

27. Lopez Rios N, Pouliot P, Papoutsis K, Foias A, Stikov N, Lesage F, Dehaes M, Cohen-Adad J (2018) Design and construction of an optimized transmit/receive hybrid birdcage resonator to improve full body images of mediumsized animals in 7T scanner. PLoS One 13(2): e0192035. https://doi.org/10.1371/journal. pone. 0192035

28. Chang D, Wang YC, Xu TT, Peng XG, Cai Y, Wang L, Bai YY, Ju S (2017) Noninvasive identification of renal hypoxia in experimental myocardial infarctions of different sizes by using BOLD MR imaging in a mouse model. Radiology 286(1):129-139. https://doi.org/10. $1148 /$ radiol.2017161998

29. Wallner BK, Edelman RR, Bajakian RL, Kleefield J, Atkinson DJ, Mattle HP (1990) Signal normalization in surface-RF coil MR imaging. Am J Neuroradiol 11(6):1271-1272

30. Hayes CE, Axel L (1985) Noise performance of surface RF coils for magnetic resonance imaging at 1.5 T. Med Phys 12(5):604-607. https://doi.org/10.1118/1.595682

31. Garwood M, DelaBarre L (2001) The return of the frequency sweep: designing adiabatic pulses for contemporary NMR. J Magn Reson 153 (2):155-177. https://doi.org/10.1006/jmre. 2001.2340 
32. Hueper K, Schmidbauer M, Thorenz A, Bräsen JH, Gutberlet M, Mengel M, Hartung D, Chen $\mathrm{R}$, Meier M, Haller $\mathrm{H}$, Wacker $\mathrm{F}$, Rong S, Gueler F (2017) Longitudinal evaluation of perfusion changes in acute and chronic renal allograft rejection using arterial spin labeling in translational mouse models. J Magn Reson Imaging 46(6):1664-1672. https://doi.org/10.1002/jmri.25713

33. Gareis D, Wichmann T, Lanz T, Melkus G, Horn M, Jakob PM (2007) Mouse MRI using phased-array RF coils. NMR Biomed 20 (3):326-334. https://doi.org/10.1002/nbm. 1156

34. Zubkov M, Hurshkainen AA, Brui EA, Glybovski SB, Gulyaev V, Anisimov NV, Volkov DV, Pirogov YA, Melchakova IV (2018) Small-animal, whole-body imaging with metamaterial-inspired RF coil. NMR Biomed 31:e3952. https://doi.org/10.1002/nbm. 3952

35. Hoult DI, Lauterbur PC (1979) The sensitivity of the zeugmatographic experiment involving human sample. J Magn Reson 34(2):425-433. https://doi.org/10.1016/0022-2364(79) 90019-2

36. Edelstein WA, Glover GH, Hardy CJ, Redington RW (1986) The intrinsic signal-to-noise ratio in NMR imaging. Magn Reson Med 3 (4):604-618. https://doi.org/10.1002/ mrm.1910030413

37. Marques JP, Simonis FFJ, Webb AG (2019) Low-field MRI: an MR physics perspective. J Magn Reson Imaging 49:1528-1542. https:// doi.org/10.1002/jmri.26637

38. Kovacs H, Moskau D, Spraul M (2005) Cryogenically cooled probes-a leap in NMR technology. Prog Nucl Magn Reson Spectrosc 46:131-155. https://doi.org/10.1016/j. pnmrs.2005.03.001

39. Ratering D, Baltes C, Nordmeyer-Massner J, Marek D, Rudin M (2008) Performance of a $200-\mathrm{MHz}$ cryogenic RF probe designed for MRI and MRS of the murine brain. Magn Reson Med 59:1440-1447. https://doi.org/ $10.1002 / \mathrm{mrm} .21629$

40. Baltes C, Radzwill N, Bosshard S, Marek D, Rudin M (2009) Micro MRI of the mouse brain using a novel $400 \mathrm{MHz}$ cryogenic quadrature RF probe. NMR Biomed 22:834-842. https://doi.org/10.1002/nbm.1396

41. Xie L, Layton AT, Wang N, Larson PEZ, Zhang JL, Lee VS, Liu C, Johnson GA (2016) Dynamic contrast-enhanced quantitative susceptibility mapping with ultrashort echo time MRI for evaluating renal function. Am J Physiol Ren Physiol 310(2):F174-F182. https://doi.org/10.1152/ajprenal.00351. 2015

42. Poirier-Quinot M, Ginefri JC, Girard O, Robert P, Darrasse L (2008) Performance of a miniature high-temperature superconducting (HTS) surface RF coil for in vivo microimaging of the mouse in a standard $1.5 \mathrm{~T}$ clinical wholebody scanner. Magn Reson Med 60 (4):917-927. https://doi.org/10.1002/ mrm.21605

43. Hu R, Kleimaier D, Malzacher M, Hoesl MAU, Paschke NK, Schad LR (2019) $\mathrm{X}$-nuclei imaging: current state, technical challenges, and future directions. J Magn Reson Imaging 5l(2):355-376. https://doi.org/10. $1002 /$ jmri.26780

44. Nagel AM, Umathum R, Rösler MB, Ladd ME, Litvak I, Gor'kov PL, Brey WW, Schepkin VD $(2016){ }^{39} \mathrm{~K}$ and ${ }^{23} \mathrm{Na}$ relaxation times and MRI of rat head at 21.1T. NMR Biomed 29:759-766. https://doi.org/10.1002/nbm. 3528

45. Schepkin VD, Brey WW, Gor'kov PL, Grant SC (2010) Initial in vivo rodent sodium and proton MR imaging at 21.1 T. Magn Reson Imaging 28(3):400-407. https://doi.org/10. 1016/j.mri.2009.10.002

46. Schepkin VD, Elumalai M, Kitchen JA, Qian C, Gor'kov PL, Brey WW (2014) In vivo chlorine and sodium MRI of rat brain at 21.1 T. MAGMA 27:63-70. https://doi.org/10. 1007/s10334-013-0387-2

47. Waiczies S, Rosenberg JT, Kuehne A, Starke L, Ramos Delgado P, Millward JM, Prinz C, Periquito J, Pohlmann A, Niendorf T (2019) Fluorine $19 \mathrm{MRI}$ at $21.1 \mathrm{~T}$ : enhanced spin-lattice relaxation of perfluorol 5 crown 5 ether and sensitivity as demonstrated in ex vivo murine neuroinflammation. MAGMA 32:37-49. https://doi. org/10.1007/s10334-018-0710-z

48. Waiczies S, Millward JM, Starke L, Ramos Delgado P, Huelnhagen T, Prinz C, Marek D, Wecker D, Wissmann R, Koch S, BoehmSturm P, Waiczies H, Niendorf T, Pohlmann A (2017) Enhanced fluorine-19 MRI sensitivity using a cryogenic radiofrequency probe: technical developments and ex vivo demonstration in a mouse model of neuroinflammation. Sci Rep 7:9808. https://doi.org/10.1038/ s41598-017-09622-2

49. Sack M, Wetterling F, Sartorius A, Ende G, Weber-Fahr W (2014) Signal-to-noise ratio of a mouse brain ${ }^{13} \mathrm{C}$ CryoProbe ${ }^{\mathrm{TM}}$ system in comparison with room temperature coils: spectroscopic phantom and in vivo results. NMR Biomed 27:709-715. https://doi.org/10. $1002 / \mathrm{nbm} .3110$ 
50. Boska MD, Meyerhoff DJ, Twieg DB, Karczmar GS, Matson GB, Weiner MW (1990) Image-guided ${ }^{31} \mathrm{P}$ magnetic resonance spectroscopy of normal and transplanted human kidneys. Kidney Int 38:294-300. https://doi. org/10.1038/ki.1990.199

51. Klemm A, Rzanny R, Fünfstück R, Werner W, Schubert J, Kaiser WA, Stein G (1998) ${ }^{31}$ P-magnetic resonance spectroscopy $\left({ }^{31} \mathrm{P}\right.$-MRS $)$ of human allografts after renal transplantation. Nephrol Dial Transplant 13 (12):3147-3152. https://doi.org/10.1093/ ndt/13.12.3147

52. Maril N, Margalit R, Mispelter J, Degani H (2004) Functional sodium magnetic resonance imaging of the intact rat kidney. Kidney Int 65:927-935. https://doi.org/10.1111/j. 1523-1755.2004.00475.x

53. Kalayciyan R, Wetterling F, Neudecker $S$, Haneder S, Gretz N, Schad LR (2013) Bilateral kidney sodium-MRI: enabling accurate quantification of renal sodium concentration through a two-element phased array system. J Magn Reson Imaging 38(3). https://doi.org/10. 1002/jmri.24024

54. Pohlmann A, Schreiber A, Ku MC, Waiczies H, Kox S, Kettritz R, Waiczies S, Niendorf T (2014) Assessment of renal inflammatory cell infiltration in a murine ANCA-induced glomerulonephritis model by 19F-MRI. In: Joint Annual Meeting ISMRM-ESMRMB 2014, Milan, Italy. https://doi.org/10.13140/RG. 2.1.4143.4326

55. Ruiz-Cabello J, Barnett BP, Bottomley PA, Bulte JWM (2011) Fluorine $\left({ }^{19} \mathrm{~F}\right)$ MRS and MRI in biomedicine. NMR Biomed 24 (2):114-129. https://doi.org/10.1002/nbm. 1570

56. Hitchens TK, Ye Q, Eytan DF, Janjic JM, Ahrens ET, Ho C (2011) ${ }^{19} \mathrm{~F}$ MRI detection of acute allograft rejection with in vivo perfluorocarbon labeling of immune cells. Magn
Reson Med 65(4):1144-1153. https://doi. org $/ 10.1002 / \mathrm{mrm} .22702$

57. Hu L, Chen J, Yang X, Senpan A, Allen JS, Yanaba N, Caruthers SD, Lanza GM, Hammerman MR, Wickline SA (2014) Assessing intrarenal nonperfusion and vascular leakage in acute kidney injury with multinuclear ${ }^{1} \mathrm{H} /{ }^{19} \mathrm{~F}$ MRI and perfluorocarbon nanoparticles. Magn Reson Med 71:2186-2196. https://doi.org/10.1002/mrm.24851

58. Pedersen $M$, Ursprung $S$, Jensen JD, Jespersen B, Gallagher F, Lautsten C (2019) Hyperpolarised ${ }^{13} \mathrm{CMRI}$ metabolic and functional imaging: an emerging renal MR diagnostic modality. MAGMA 33(1):23-32. https:// doi.org/10.1007/s10334-019-00801-y

59. Gordon CJ (2017) The mouse thermoregulatory system: its impact on translating biomedical data to humans. Physiol Behav 179:55-66. https://doi.org/10.1016/j.physbeh.2017.05. 026

60. Reimann HM, Hentschel J, Marek J, Huelnhagen T, Todiras M, Kox S, Waiczies S, Hodge R, Bader M, Pohlmann A, Niendorf T (2016) Normothermic mouse functional MRI of acute focal thermostimulation for probing nociception. Sci Rep 6:17230. https://doi. org/10.1038/srepl7230

61. Hitt BA, Mazze RI, Cook TL, Beppu WJ, Kosek JC (1977) Thermoregulatory defect in rats during anesthesia. Anesth Analg 56 (1):9-15. $\quad$ https://doi.org/10.1213/ 00000539-197701000-00006

62. Niles DJ, Gordon JW, Fain SB (2016) Effect of anesthesia on renal $\mathrm{R}_{2}{ }^{*}$ measured by blood oxygen level-dependent MRI. NMR Biomed 28(7):811-817. https://doi.org/10.1002/ nbm.3314

63. Nikken JJ, Krestin GP (2007) MRI of the kidney-state of the art. Eur Radiol 17 (11):2780-2793. https://doi.org/10.1007/ s00330-007-0701-3

Open Access This chapter is licensed under the terms of the Creative Commons Attribution 4.0 International License (http://creativecommons.org/licenses/by/4.0/), which permits use, sharing, adaptation, distribution and reproduction in any medium or format, as long as you give appropriate credit to the original author(s) and the source, provide a link to the Creative Commons license and indicate if changes were made.

The images or other third party material in this chapter are included in the chapter's Creative Commons license, unless indicated otherwise in a credit line to the material. If material is not included in the chapter's Creative Commons license and your intended use is not permitted by statutory regulation or exceeds the permitted use, you will need to obtain permission directly from the copyright holder. 